\title{
Erratum: Majorana-Hubbard model on the square lattice [Phys. Rev. B 96, 125121 (2017)]
}

\author{
Ian Affleck, Armin Rahmani, and Dmitry Pikulin \\ (Received 17 December 2017; published 3 January 2018)
}

DOI: 10.1103/PhysRevB.97.039901

Figure 8 and its caption should be replaced by the one below.

In addition, the second sentence below Eq. (6.14) should read "The terms in the effective Hamiltonian which break the U(1) (and Lorentz) symmetry are of dimension six or higher, more irrelevant than the relativistic interactions term."

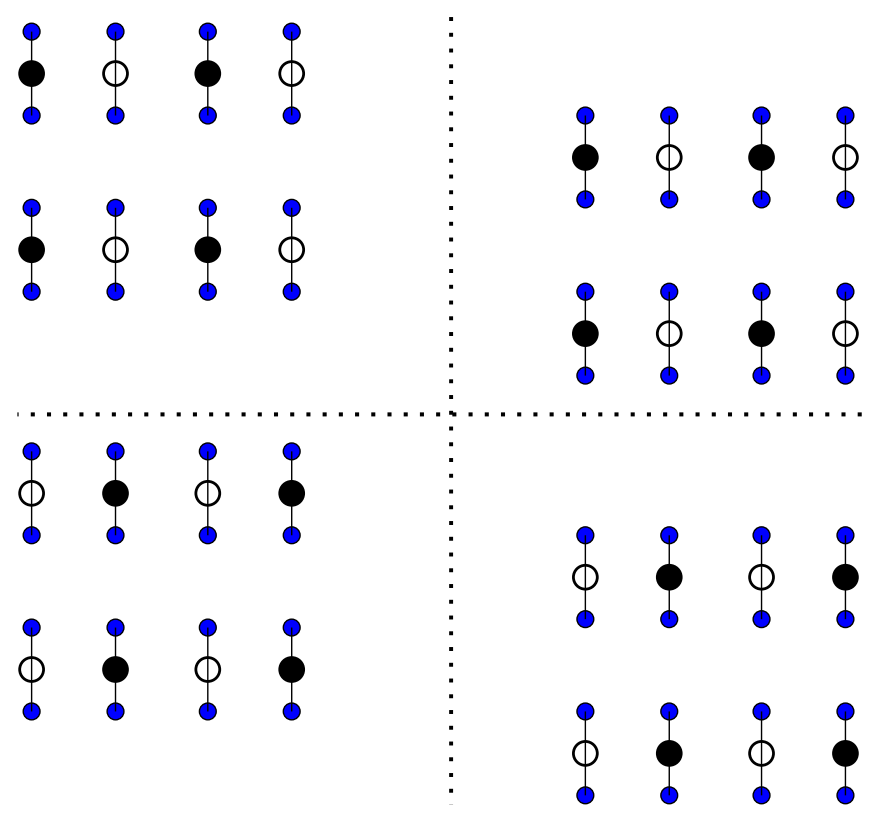

FIG. 8. Sketch of four mean-field ground states occurring for $g<0$. The blue dots represent lattice sites. The large circles appear on bonds on which the two Majorana modes combine to form Dirac fermions with the filled or the empty circles corresponding to the Dirac level being occupied or empty. In addition, there are four equivalent ground states with Dirac fermions occurring on horizontal bonds. 